\title{
Proton pump inhibitors and clopidogrel - hazardous drug interaction or hazardous interpretation of data?
}

\author{
Paul Moayyedi PhD MRCP FRCP ${ }^{1}$, Daniel C Sadowski MD FRCPC ${ }^{2}$
}

$\mathrm{G}^{\mathrm{a}}$ astroenterologists frequently prescribe proton pump Jinhibitors (PPIs) for the prevention of nonsteroidal antiinflammatory drug-induced upper gastrointestinal (GI) bleeding. There is good scientific evidence to support this treatment strategy (1). It is increasingly recognized that antiplatelet agents such as clopidogrel are also associated with increased rates of GI bleeding, similar to those reported with cardioprotective doses of acetylsalicylic acid (ASA) (2). Indeed, a randomized controlled trial (3) has demonstrated that the risk of GI bleeding is higher in patients taking clopidogrel compared with those taking ASA plus a PPI. When administered in combination with ASA, clopidogrel is particularly potent in increasing the risk for upper GI bleeding (RR of bleeding 15.2; 95\% CI 4.1 to 56.5) (4). Because of this, PPIs are prescribed with clopidogrel in this situation in the belief that their administration will reduce bleeding. Until recently, the safety of this therapeutic manoeuvre has not been questioned.

It is well known in the cardiovascular community that the antiplatelet effect of clopidogrel varies from patient to patient, and that reduced platelet inhibition by clopidogrel is associated with an increased risk for cardiac events (5). The mechanisms underlying clopidogrel resistance are controversial and may relate to heterogeneity in clopidogrel metabolism. Clopidogrel is a prodrug that requires metabolism by cytochrome P450 to an active form. One isoenzyme potentially critical in this step is cytochrome P450 2C19 (CYP2C19). It has been demonstrated that this critical enzyme can be inhibited by PPIs and that reduced patient responsiveness to clopidogrel may be associated with PPI use. An example of this drug-drug interaction is seen in the Omeprazole CLopidogrel Aspirin (OCLA) study (6). One hundred twenty-four consecutive patients undergoing coronary artery stent implantation were randomly assigned to clopidogrel plus omeprazole $(20 \mathrm{mg} /$ day $)$ or clopidogrel plus placebo. The effect of clopidogrel on platelet function was assessed by using the platelet phosphorylated vasodilatorstimulated phosphoprotein assay at day 7 . The study found that the omeprazole group had a significantly decreased clopidogrel inhibitory effect on platelet function. This report has prompted the United States Food and Drug Administration to request additional studies from the manufacturers of clopidogrel (sanofi-aventis, Bristol-Myers Squibb) to further characterize this potential interaction. In addition to the OCLA trial, two large observational studies $(7,8)$ presented in abstract form have suggested that PPIs may attenuate the beneficial effects of clopidogrel. However, these studies have several shortcomings and a joint comment by the American College of Cardiology (ACC), the American Heart Association (AHA) and the
American College of Gastroenterology (ACG) stated that "In the interest of patient safety, the AHA/ACC and the ACG advise that patients who are currently taking these medications should not change their medication regimen unless advised by their health care provider" $(9,10)$.

A new Canadian study by Juurlink et al (10) examined hospital discharge data after treatment for myocardial infarction. The investigators found that readmission rates for cardiovascular events within 90 days were statistically higher in patients taking PPIs and clopidogrel. This interaction was not demonstrated with pantoprazole. Similar observations were made in a recently published American study (11) that was previously available only in abstract form. Should these new data change the recommendation by ACC/AHA and ACG? Should we avoid using PPI therapy in patients taking clopidogrel or at least switch them to pantoprazole?

The Canadian (10) and American (11) studies are difficult to interpret because the increase in the RR of cardiovascular events for patients taking PPIs was very modest. Because these studies relied on provincial or Veterans Affairs' retrospective databases, the authors were unable to control for important confounding factors. Studies (12) have shown that patients at high risk for upper GI bleeding (and therefore more likely to be prescribed PPI therapy) are also at higher risk of mortality from cardiovascular events. The results seen in these observational studies may simply be due to a greater tendency to prescribe prophylactic PPIs to patients at higher risk of cardiovascular events. Therefore, it would be important to control for predictors of recurrent myocardial infarction such as left ventricular function, smoking status, ASA use and blood pressure. In both studies, the control and case groups had marked differences in important comorbid health factors, with those taking PPIs having a higher prevalence of renal disease, cancer, chronic obstructive pulmonary disease, congestive heart failure, low left ventricular ejection fraction, previous myocardial infarction, previous coronary artery bypass surgery and diabetes mellitus. The authors performed the appropriate analyses to control for these imbalances but statistics cannot control for unknown or unmeasured confounders that may also exist between the two groups. Given the differences both studies found between cases and controls, it is likely that further imbalances exist and the results could simply be due to residual confounding. To demonstrate the effect of these confounders, a randomized controlled trial (13) of clopidogrel versus placebo stratified for PPI use found that patients given PPIs were more likely to have a cardiovascular event at one year compared with subjects not taking PPIs. Clopidogrel, however, reduced the incidence of

${ }^{1}$ Gastroenterology Division, McMaster University Health Sciences Centre, Hamilton, Ontario; ${ }^{2}$ Division of Gastroenterology, Royal Alexandra Hospital, Edmonton, Alberta

Correspondence: Dr Daniel C Sadowski, 331 Community Services Centre, Royal Alexandra Hospital, 10240 Kingsway Avenue, Edmonton, Alberta T5H 3V9. Telephone 780-735-6837, fax 780-735-5650, e-mail dan.sadowski@ualberta.ca

Received and accepted for publication March 10, 2009 
cardiovascular events at one year compared with placebo, regardless of whether patients were taking a PPI.

Are there other factors at play that may provide an alternative explanation to the PPI-clopidogrel interaction theory? Recently, the existence of reduced-function CYP2C19 alleles has been characterized (14). It was shown that carriers of a CYP2C19 reduced-function allele have significantly lower levels of the active metabolite of clopidogrel, diminished platelet inhibition and a higher rate of major adverse cardiovascular events, including stent thrombosis, than do noncarriers. To date, there are no published reports of the interaction between these alleles and PPIs. The distribution of these reducedfunction alleles in the general population is unknown but preliminary evidence suggests that they may be present in 30\% of Caucasians and in up to 55\% of East Asians (15).

Is there a difference between PPIs in their ability to inhibit CYP2C19? Juurlink et al (10) suggest that pantoprazole does not significantly inhibit CYP2C19. However, a systematic review (16) evaluating the impact of PPIs on cytochrome P450 suggests that the data are heterogeneous and the picture is far from clear. As well, a recent Austrian study (17) of 300 patients undergoing coronary stent insertion found no effect of either pantoprazole or esomeprazole on platelet inhibition by clopidogrel. Esomeprazole was not evaluated by Juurlink et al (10) because this drug was not captured by the Ontario Health Insurance Plan database. Furthermore, from an epidemiological perspective, the data regarding pantoprazole are not

\section{REFERENCES}

1. Rostom A, Moayyedi P, Hunt R, et al; for the Canadian Association of Gastroenterology Consensus Group. Canadian consensus guidelines on long-term nonsteroidal anti-inflammatory drug therapy and the need for gastroprotection: Benefits versus risks. Aliment Pharmacol Ther 2009;29:481-96.

2. Bhatt DL, Scheiman J, Abraham NS, et al. ACCF/ACG/AHA 2008 Expert consensus document on reducing gastrointestinal risks of antiplatelet therapy and NSAID use: A report of the American College of Cardiology Foundation Task Force on clinical expert consensus documents. Circulation 2008;118:1894-1909.

3. Chan FK, Ching JY, Hung LC, et al. Clopidogrel versus aspirin and esomeprazole to prevent recurrent ulcer bleeding. $\mathrm{N}$ Engl J Med 2005;352:238-44.

4. Lanas A, Garcia-Rodriguez LA, Arroyo MP, et al. Risk of upper gastrointestinal ulcer bleeding associated with selective cyclooxygenase- 2 inhibitors, traditional non-steroidal non-aspirin antiinflammatory drugs, aspirin and combinations. Gut 2006;55:1731-8.

5. Matetzky S, Shenkman B, Guetta V, et al. Clopidogrel resistance is associated with increased risk of recurrence of atherothrombotic events in patients with acute myocardial infarction. Circulation 2004;109:3171-5.

6. Gilard M, Arnaud B, Cornily JC, et al. Influence of omeprazole on the antiplatelet action of clopidogrel associated with aspirin: A randomized, double-blind OCLA (Omeprazole Clopidogrel Aspirin) Study. J Am Coll Cardiol 2008;51:256-60.

7. Ho MP, Maddox TM, Wang L, et al. Proton pump inhibitors may attenuate the benefits of clopidogrel among ACS patients: Empirical evidence from 3,311 ACS patients. Circulation 2008;118:S1165. (Abst)

8. Aubert RE, Epstein RS, Teagarden JR, et al. Proton pump inhibitors effect on clopidogrel effectiveness: The clopidogrel medco outcomes study. Circulation 2008;118:S815. (Abst)

9. American College of Cardiology (ACC)/American College of Gastroenterology (ACG)/American Heart Association (AHA) as statistically signficant as Juurlink et al (10) claim. When conducting subgroup analyses, it is important not to find just one subgroup that is (or is not) statistically significant for the outcome of interest. The conservative statistical approach is to determine whether one subgroup is significantly different from the other (ie, if there is significant heterogeneity between groups). When this analysis was conducted, there was no significant difference between pantoprazole and the other PPIs $\left(\chi^{2}=2.99\right.$, degrees of freedom $=1 ; \mathrm{P}=0.08$ ).

In light of the above, should PPI prophylaxis of GI bleeding for patients taking ASA and clopidogrel be curtailed? Given the ambiguity in the current level of evidence, we believe that the conclusion reached by the ACC/AHA and ACG remains valid. The United States Food and Drug Administration have also maintained that there is insufficient evidence regarding the interaction between clopidogrel and PPIs, and have highlighted the need for further studies (18). The data are far from clear and multiple confounders require a careful reappraisal of the literature. In particular, some of the previous investigations, such as the OCLA study, will need to be repeated with a subgroup analysis based on reduced-function CYP2C19 allele status. GI bleeding in the setting of ASA and clopidogrel is often severe and is frequently associated with adverse outcomes in groups with pre-existing cardiovascular disease. Discontinuation of PPI prophylaxis for these patients, given the current shaky level of evidence, is premature at best and hazardous at worst.
Joint Comment on Studies Regarding Possible Interaction of Clopidogrel and Proton Pump Inhibitors. November 11, 2008. $<$ http://americanheart.mediaroom.com/index.php?s=43\&item $=611>$ (Version current at February 23, 2009).

10. Juurlink DN, Gomes T, Ko DT, et al. A population-based study of the drug interaction between PPIs and clopidogrel. CMAJ 2009. (In press)

11. Ho MP, Maddox TM, Wang L, et al. Risk of adverse outcomes associated with concomitant use of clopidogrel and proton pump inhibitors following acute coronary syndrome. JAMA 2009;301:937-44.

12. Wang TY, Chen AY, Roe MT, et al. Balancing mortality benefit vs. bleeding risk for intensive antiplatelet therapy among acute coronary syndrome patients: Insights from the CRUSADE registry. Circulation 2008;118:S916.

13. Dunn SP, Macaulay TE, Brennan DM, et al. Baseline proton pump inhibitor use is associated with increased cardiovascular events with and without the use of clopidogrel in the CREDO trial. Circulation 2008;118:S815. (Abst)

14. Mega JL, Close SL, Wiviott SD, et al. Cytochrome P-450, polymorphisms and response to clopidogrel. N Engl J Med 2009;360:354-62.

15. Desta Z, Zhao X, Shin JG, et al. Clinical significance of the cytochrome P-450 2C19 genetic polymorphism. Clin Pharmacokinet 2002;41:913-58.

16. Chong E, Ensom MH. Pharmacogenetics of the proton pump inhibitors: A systematic review. Pharmacotherapy 2003;23:460-71.

17. Li X-Q, Andersson TB, Ahlstrom M, Weidolf L. Comparison of inhibitory effects of the proton pump-inhibiting drugs omeprazole, esomeprazole, lansoprazole, pantoprazole and rabeprazole on human cytochrome P450 activities. Drug Metab Dispos 2004;32:821-7.

18. Food and Drug Administration. Early communication about an ongoing safety review of clopidogrel bisulfate (marketed as Plavix). $<$ http://www.fda.gov/cder/drug/early_comm/clopidogrel_bisulfate. $\mathrm{htm}>($ Version current at March 8, 2009). 


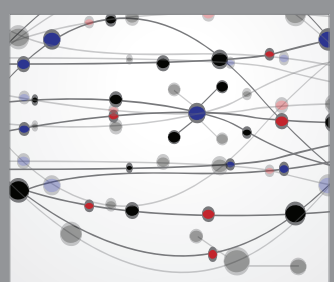

The Scientific World Journal
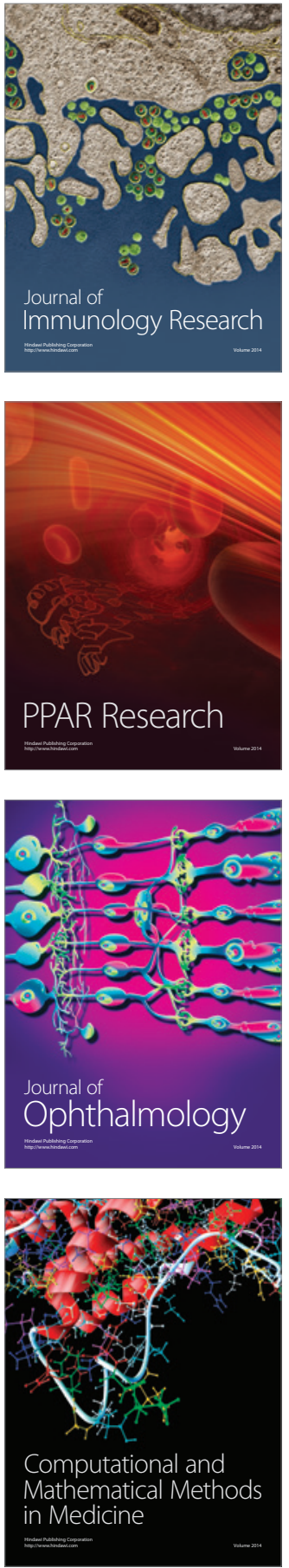

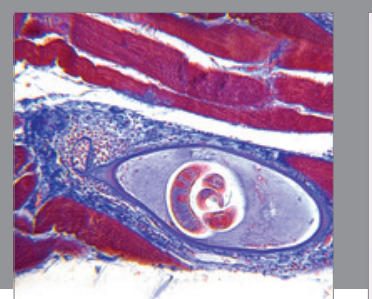

Gastroenterology Research and Practice

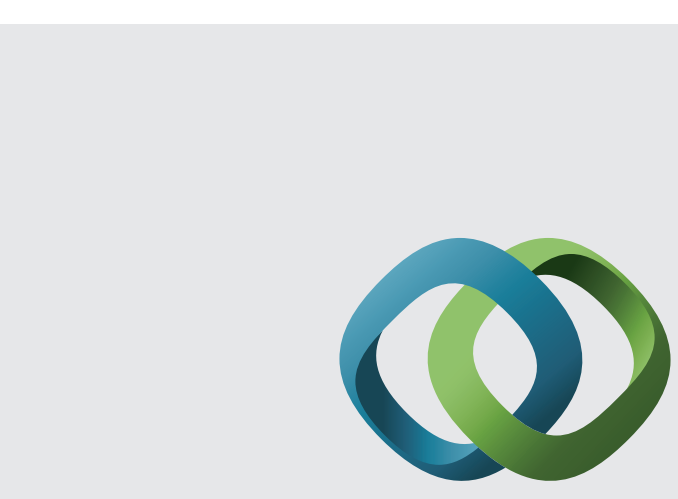

\section{Hindawi}

Submit your manuscripts at

http://www.hindawi.com
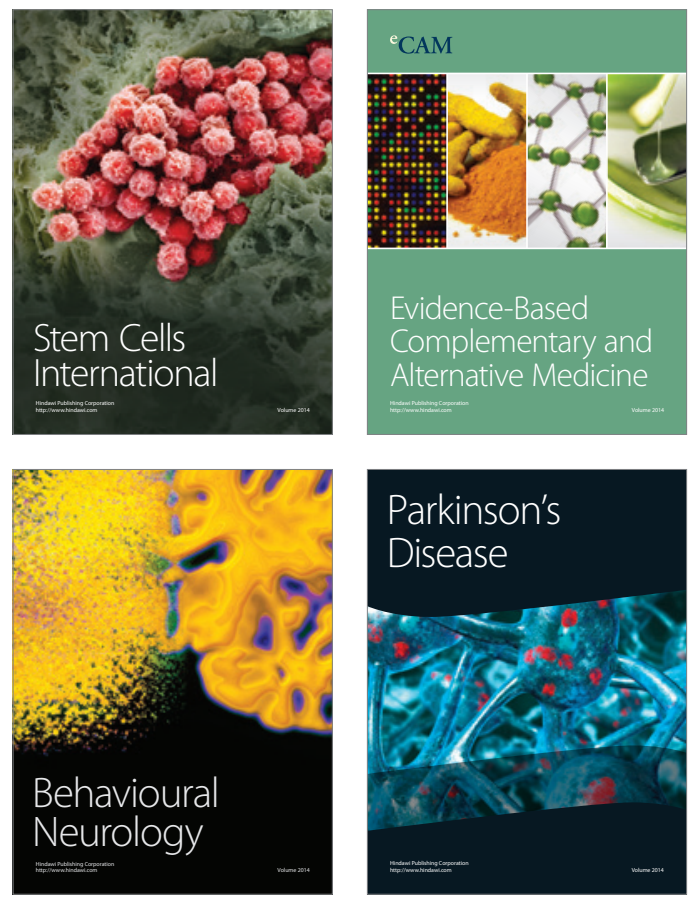
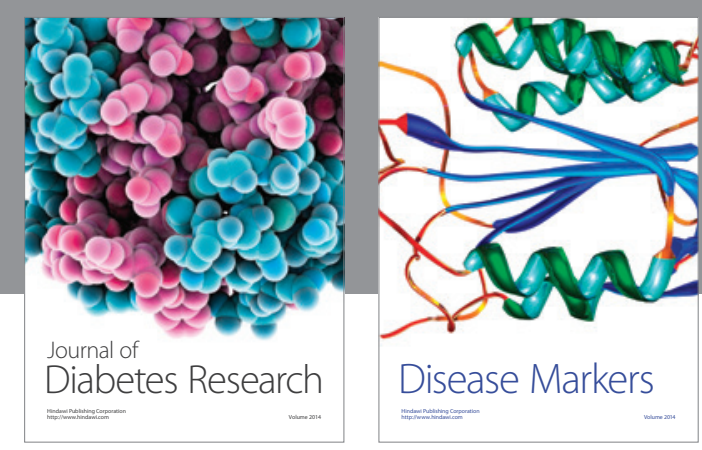

Disease Markers
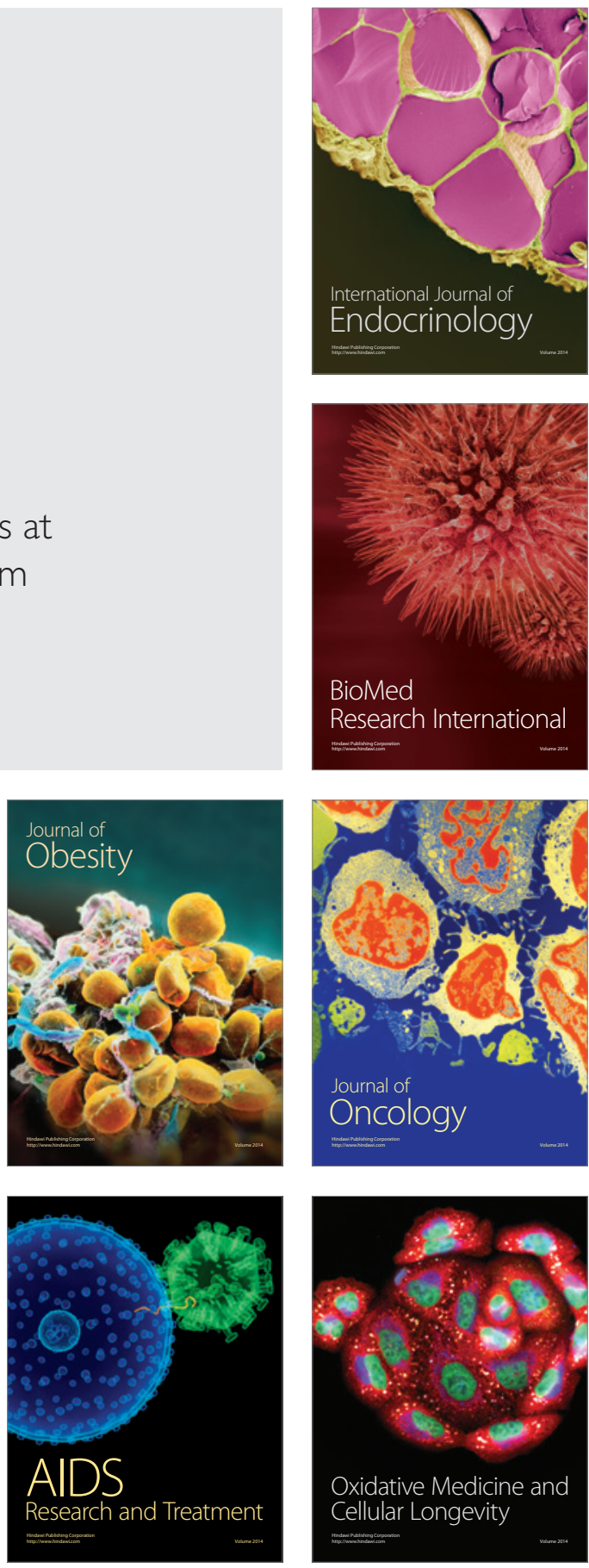